\title{
Axillary fibromatosis with radiological- pathological correlation: a case report and literature review
}

\author{
Flávia Sprenger ${ }^{1}$, Sofia Tokars Kluppel ${ }^{2}$, Valmir Vicente Filho ${ }^{2^{*}} \mathbb{D}$, Ana Carolina Staats ${ }^{2}$, \\ Raul Alberto Anselmi Junior ${ }^{3}$ and Maria Helena Louveira ${ }^{1}$
}

\begin{abstract}
Background: The term fibromatosis refers to a spectrum of soft tissue tumors resulting from the unbridled proliferation of fibroblasts, with high infiltrative power. It is a rare neoplasm, with an incidence of about 3 cases per million. Most common sites include the abdominal wall and cavity, chest wall, scapular area, and the limbs, rarely affecting the axilla. Imaging plays a major role in surgical planning and clinical follow-up. Differential diagnosis with other soft tissue tumors may be arduous, but a correct evaluation is fundamental.

Case presentation: A 33-year-old case is reported for the evaluation of a palpable hardened and immobile left axillary nodule. The ultrasound reveals a solid, elongated, heterogeneous, poorly delimited, infiltrative lesion observed in the left axillary hollow, with no detectable flow in the color Doppler mode. At magnetic resonance imaging, the same elongated and expansive lesion was better defined, revealing its irregular contours, alternating areas of hypo- and hyperintense on T2, heterogeneous enhancement, and no signs of signal decay on in- and outof-phase sequences. Due to its growth and local invasion potential, surgical excision was performed. The microscopic analysis showed long and uniform spindle cell fascicles, with clear cytoplasm and wavy nuclei, arranged in different directions, included in collagen stroma. Immunohistochemistry was positive for nuclear betacatenin, confirming the diagnosis of fibromatosis.

Conclusion: Imaging methods reflect the heterogeneous nature of the lesion. This study demonstrates the importance of using a multidisciplinary approach in addition to imaging tests and histopathological study for better diagnosis and therapeutic planning. The high infiltrative power always must be reminded of, since it affects young patients and diagnosis delay can lead to mutilating surgeries.
\end{abstract}

Keywords: Fibromatosis, Armpit, Desmoid tumor, Imaging methods, Case report

\section{Background}

The term fibromatosis refers to a spectrum of soft tissue tumors resulting from the unbridled proliferation of fibroblasts, without metastatic potential, but locally invasive [1]. It is a rare neoplasm, with an incidence of about 3 cases per million, representing only $0.03 \%$ of all neoplasms and less than $3 \%$ of all soft tissue tumors [2, 3].

\footnotetext{
*Correspondence: valmirvicentef@gmail.com

${ }^{2}$ Health Science Sector, Hospital de Clínicas da Universidade Federal do

Paraná, Curitiba, Paraná, Brazil

Full list of author information is available at the end of the article
}

However, it makes differential diagnosis with important and aggressive soft tissue tumors, especially in unusual places, such as the armpits.

The aim of this study is to present the case of a 33year-old female patient, who was referred for the evaluation of a palpable, hardened, immobile nodule in the region of the left armpit. The patient reported a 2month history of progressive growth. A rare case of axillary fibromatosis was diagnosed. 


\section{Case presentation}

A 33-year-old truck driver female patient referred to our service for the evaluation of a palpable, hardened, immobile left axillary nodule. She had a history of breast augmentation 5 years ago, without any other relevant medical history. Patient mentioned progressive growth in the past 2 months.

On examination, she had a palpable, hard, painless nodule adhered to her left armpit. However, she had no motor disabilities in the left upper limb. The investigation began with an ultrasound (US) study of the left axilla. A solid, elongated, heterogeneous, poorly delimited, infiltrative lesion was observed in the left axillary hollow, with no detectable flow in the color Doppler mode. The tumor had alternating echogenicity in layers and extensions that infiltrated the locoregional adipose planes, forming the tail signal along its major axis. In addition to a fatty invasion, it was not possible to establish a cleavage plan with the muscular belly of the coracobrachialis (Fig. 1).

Evaluation proceeded with a magnetic resonance imaging (MRI) study of the breasts and axillae. The same elongated and expansive lesion was better defined, revealing its irregular contours, alternating areas of hypoand hyperintense on T2, heterogeneous enhancement, and no signs of signal decay on in- and out-of-phase sequences. The tumor was located between the fibers of the third proximal of the coracobrachialis muscle belly, without a cleavage plane with some deeper fibers of the adjacent brachial biceps (Fig. 2).

No suspicious breast lesions or axillary lymph nodes were identified. The surrounding bone framework was assessed by MRI. The hypothesis of soft tissue neoplasm was then raised, given the absence of associated findings in the breasts and in relation to lymph node structures.

Patient underwent US-guided core biopsy, which revealed a fusocellular proliferation disposed on collagen bundles, suggestive of fibromatosis.

Due to its growth and local invasion potential, surgical excision was performed. Function preservation was the main goal of the surgery, since the patient's work activity requires maintaining the strength of the upper limb. Thus, the patient was monitored throughout the intraoperative period using real-time-electroneuromyography (Fig. 3).

Surgical specimen macroscopic evaluation showed an irregular, permeative, whitish, shiny, firm lesion, infiltrating the fat planes and compromising the deep surgical margin.

Microscopic analysis showed long and uniform spindle cell fascicles, with clear cytoplasm and wavy nuclei, arranged in different directions, included in collagen
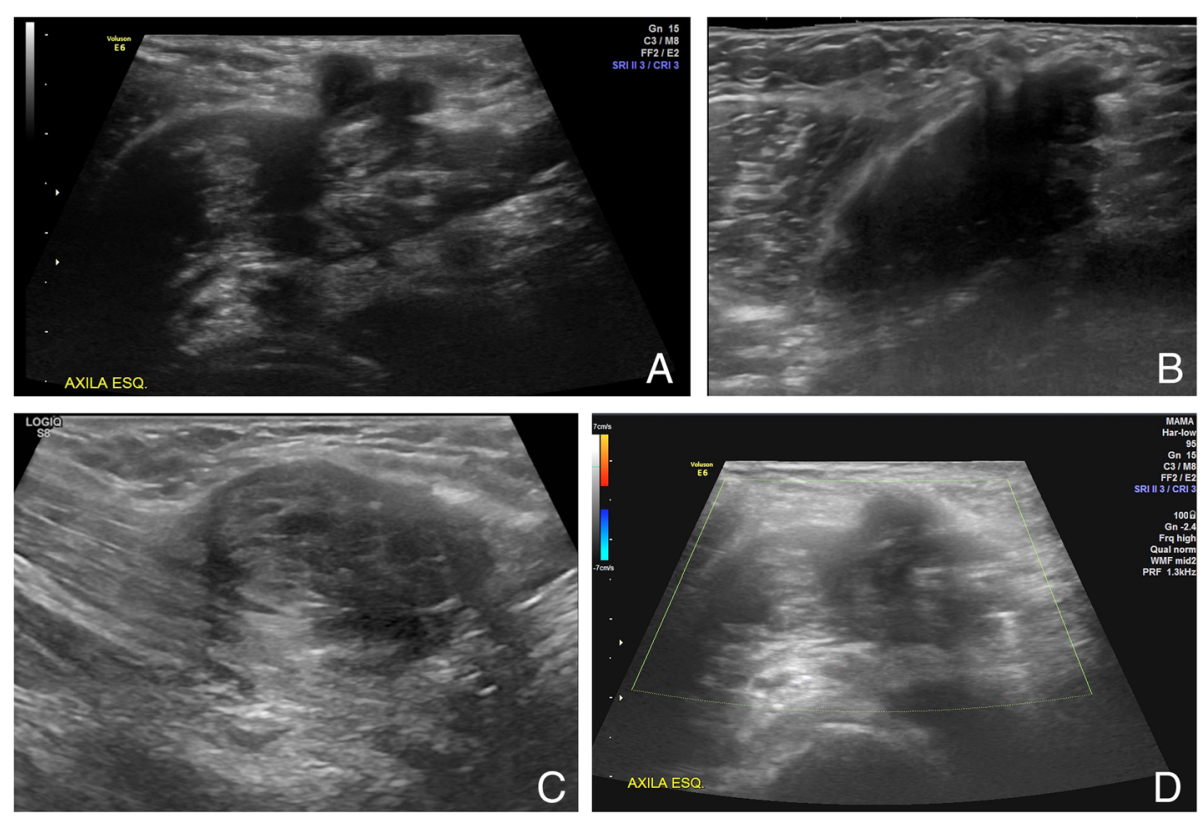

Fig. 1 US of the left axillary region. A Longitudinal image of the solid, heterogeneous, elongated, irregular lesion, with alternating echogenicities arranged in layers (pink arrow), forming the tail sign (green arrow) along the longest axis. B Extended view of the aforementioned lesion, showing digitiform extensions that extend to adjacent adipose planes (blue arrows). C Transverse image of the tumor, showing alternating echogenicity layers (pink arrow) as well as signs of invasion of the coracobrachialis muscle (white arrow). D There was no vascularity detectable in the color Doppler mode 


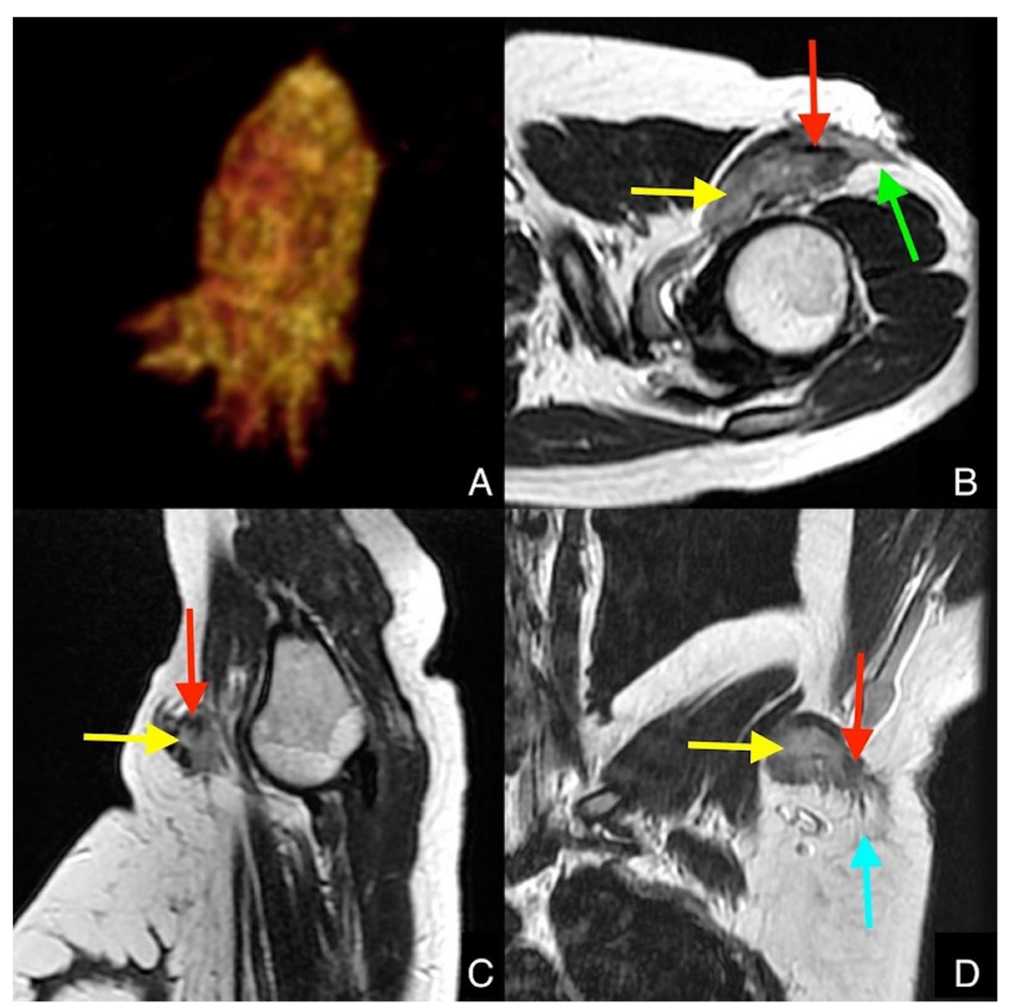

Fig. 2 MRI images of the left armpit. A Three-dimensional reconstruction with volume rendering of the lesion showing its ill-defined and infiltrative aspect. B Axial pre-contrast T2 showing a heterogeneous, irregular and poorly delimited lesion in the left axillary region, extending through the adipose planes forming the tail sign (green arrow). Hypersignal areas are noted (yellow arrow), as well as low signal bands (band signal-red arrow). C Sagittal pre-contrast T2. A yellow arrow highlights the area with the greatest signal, and a red arrow shows the hyposignal bands. D Coronal pre-contrast T2 representing once again the areas of high signal (yellow arrow) and low signal (red arrow), in addition to fine projections of the tumor to the adjacent fat planes (blue arrow)

stroma. There was an abundance of vessels, elongated vessels, and thin walls. No atypical cells or other signs of malignancy were seen, such as hyperchromasia and mitosis. Radiological-pathological correlations are exposed in Fig. 4. Immunohistochemistry was positive for nuclear beta-catenin, confirming the diagnosis of fibromatosis (Fig. 5).

The patient was discharged from the hospital in good general condition, and after histopathological confirmation of the involvement of the deep margins, given the high chances of local recurrence, the patient awaits a new MRI for a therapeutic decision between amplifying surgical margins or adjuvant with radiotherapy.

\section{Discussion}

Fibromatosis, also known as desmoid tumors [4], is a rare type of neoplasm; its estimated annual incidence is 900 patients in the USA [5]. Despite its low metastatic potential, local invasion can lead to deformities and mutilating surgeries. They result in significant psychological and economic effects on the lives of young adults $[5,6]$.
Most common sites include the abdominal wall and cavity, chest wall, scapular area, and the limbs, rarely affecting the axilla [2]. Less frequently, intraabdominal forms are associated with familial adenomatous polyposis or Gardner's syndrome, usually affecting the mesentery. It is a condition always to be within the differential diagnosis of soft tissue tumors on imaging $[1,2]$.

Risk factors include the first year after pregnancy and the use of oral contraceptives [3]. Pathophysiology relies on the excess of intracellular beta-catenin, a molecule fundamental to intercellular adhesion of mesenchymal cells [4].

Differential diagnosis include nodular fasciitis, fibroma of the tendon sheath, tumors of the neural sheath, fibroadenomas, dermatofibroma, and leiomyoma [7].

Imaging has a major impact on therapeutic and surgical planning. Aspects such as the infiltrative nature of the tumor, margins for resection, and recurrence rates are determinant in morbidity and in the impact on quality of life $[5,6]$. 

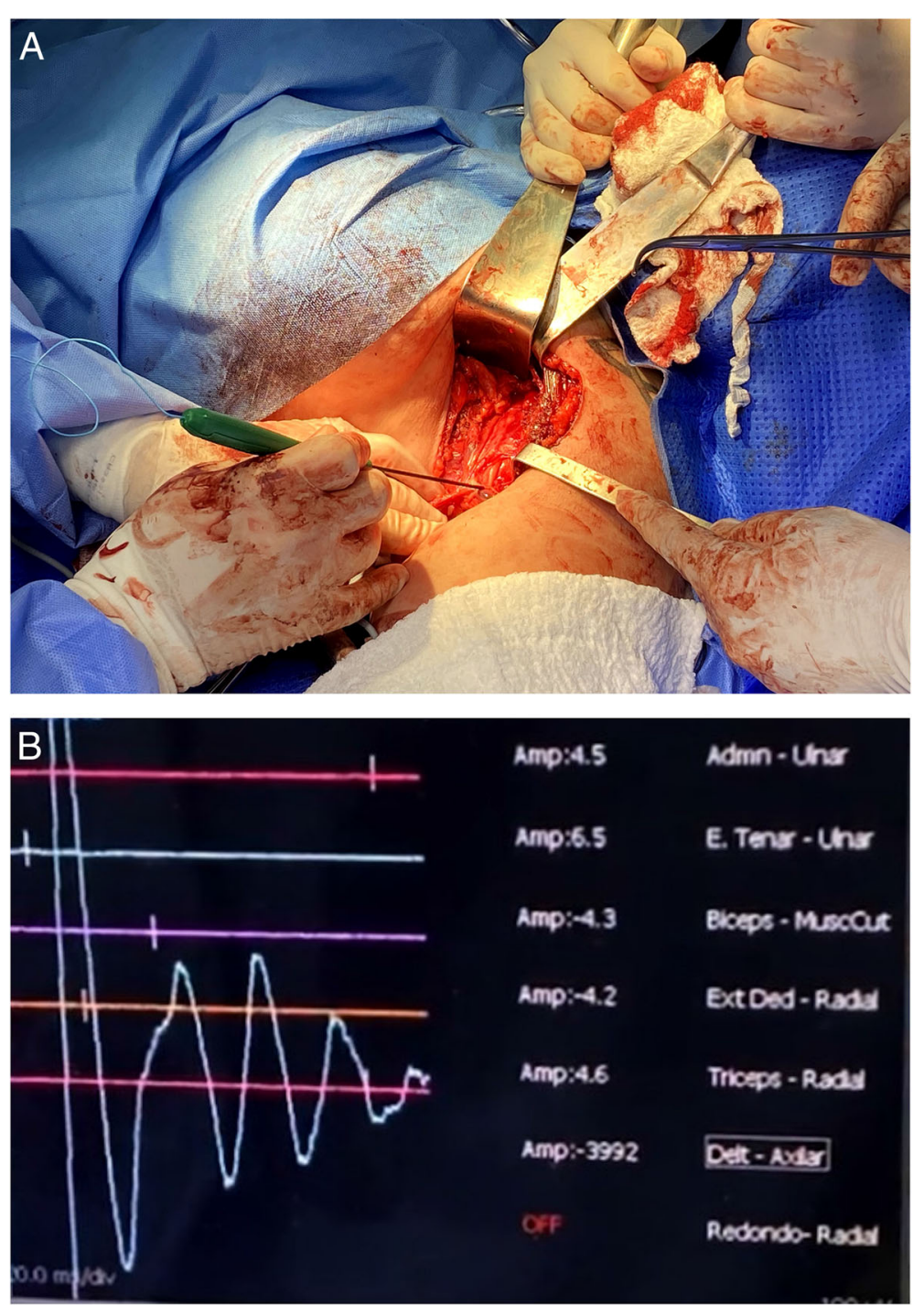

Fig. 3 Intraoperative image of function-preserving surgery with electroneuromyography in real time. A An electrostimulation pen (green arrow) stimulates a structure that raised doubts about its nervous origin (white asterisk). B During monitoring, the muscular contraction of the deltoid was detected, triggering waves (white arrows), proving that the structure in question was the axillary nerve and, therefore, should not be sectioned

US is a non-expensive and highly accessible initial method. Findings include a regular or irregular heterogeneous solid mass, circumscribed or with poorly defined margins [1]. Echogenicity is variable and closely related to histological heterogeneity. Color Doppler vascularization is also variable, more commonly absent [4].

On computerized tomography (CT), fibromatosis appears as a mass with soft tissue density, with different degrees of attenuation according to histological composition, with well-defined or ill-defined margins. A higher proportion of collagen increases tomographic density. This is particularly useful on clinical follow-up, since the increase in density reflects a good response to treatment [2].
Necrosis and calcification are not expected. Bone involvement occurs in 5 to $30 \%$ of cases, with a greater role for computed tomography in these situations [8].

MRI currently represents the imaging modality of choice in the evaluation of soft tissue injuries of the extremities and trunk [3].

MRI findings include heterogeneous iso- to hyperintense masses on T1- and T2-weighted sequences. Signal is highly variable, reflecting the proportion of cellular and fibrotic components of the tumor. The hypointense areas on T2 represent high cellularity and dense collagen, and the hyperintense areas in T2 represent low cellularity $[1,9]$.

Classical signals are described, such as the band sign, which consists of the presence of hypointense lines or 


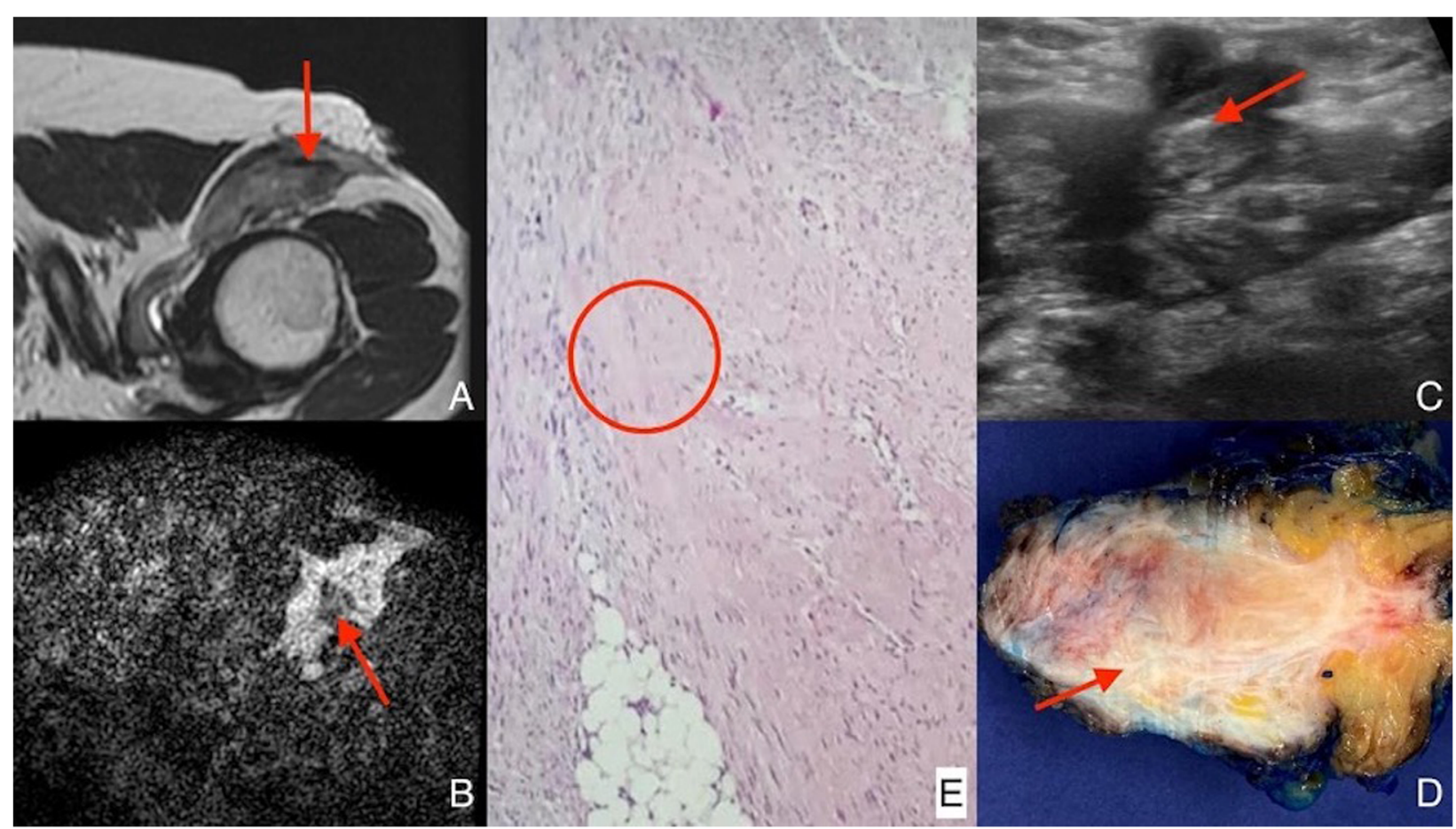

Fig. 4 Radiological-pathological correlation. The hypointense areas in T2 (red arrow in A, axial T2 pre-contrast), poor enhancement in post-contrast sequences (red arrow in B, axial T1 3D post-contrast), bands of hyperechogenicity at US (arrow red in C) and more hardened and brighter areas at macroscopy (red arrow in D) correspond to areas of cell poverty, with a predominance of fibrous stroma (red circle in E, HE 100X)

bands in $\mathrm{T} 1$ and $\mathrm{T} 2$, without enhancement; the tail signal, which like the ultrasound, reflects the infiltrative nature of the tumor, forming elongated extensions; the split fat sign, represented by a tenuous annular enhancement of the adipose planes adjacent to it, and, finally, the flame sign, which consists of linear enhancements extending across the fascial planes [4].

The main therapeutic modality is excision. Desmoid tumors are associated with high levels of recurrence, especially when surgical margins are compromised [3]. Other

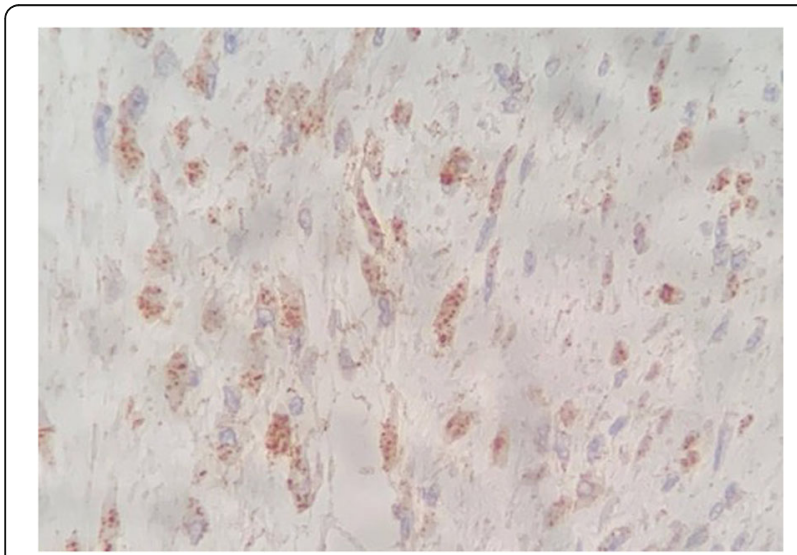

Fig. 5 Immunohistochemistry: positive for nuclear beta-catenin, the main immunohistochemistry marker of fibromatosis more modern options include radiotherapy, anti-estrogenic drugs, non-steroidal anti-inflammatory drugs, cytotoxic chemotherapy, and tyrosine kinase inhibitors [7].

Macroscopically, it is a whitish, shiny tumor, containing thick trabeculae resembling scar tissue, some arranged in a swirl, in addition to strands of fibrous tissue infiltrating the adjacent musculoadipous planes [8].

On microscopic analysis, fibromatosis presents as long and extensive fascicles of uniform spindle cells, with pale cytoplasm and wavy nuclei, included in a vast collagen stroma. Elongated vessels with thin walls and areas of micro-hemorrhage are common. Cell atypia and other signs of malignancy are not expected [5]. Immunohistochemistry is important for diagnosis, being positive for betacatenin and smooth muscle actin [10].

\section{Conclusion}

This study reinforces the importance of using a multidisciplinary approach in addition to imaging tests and histopathological study for better diagnosis and therapeutic planning for axillary fibromatosis. We revisit the classical presentation of desmoid tumors on all imaging methods and the emblematic signs, such as the tail sign and the flame sign. Imaging methods, such as US, CT, and MRI reflect the heterogeneous nature of the lesion. The high infiltrative potential must always be reminded of, since it affects young patients and diagnosis delay can lead to mutilating surgeries. 


\section{Abbreviations}

US: Ultrasound; MRI: Magnetic resonance imaging; CT: Computerized Tomography

\section{Acknowledgements}

We offer our deepest appreciation to the institution that provided technical support for the development and implementation of this study.

\section{Authors' contributions}

The authors read and approved the final manuscript. FS was responsible for the investigation of background information, curation of case information, and radiographic images, analysis, drafting, review, and editing of the manuscript. STK, WF, and ACS were responsible for the conceptualization of the case report, methodology of approaching investigation of background information, and writing of manuscript as well as reviewing/editing of the manuscript. RAAJ was responsible for the curation of intraoperative images, oversight of the investigation, and reviewing/editing of the manuscript. MHL was responsible for the investigation of background information, oversight of the investigation, curation of case information, and radiographic images, analysis, and review the manuscript.

\section{Funding}

Not applicable.

\section{Availability of data and materials}

All the data and materials were obtained from patients registered in our hospital.

\section{Declarations}

\section{Ethics approval and consent to participate}

The patient included in this study gave written consent to participate in this research.

\section{Consent for publication}

Written informed consent was obtained from the patient for publication of this case report and accompanying images.

\section{Competing interests}

The authors declare that they have no competing interests.

\section{Author details}

'Department of Radiology, Hospital de Clínicas da Universidade Federal do Paraná, Curitiba, Brazil. ${ }^{2}$ Health Science Sector, Hospital de Clínicas da Universidade Federal do Paraná, Curitiba, Paraná, Brazil. ${ }^{3}$ Department of Surgery, Hospital de Clínicas da Universidade Federal do Paraná, Curitiba, Paraná, Brazil.

Received: 14 May 2021 Accepted: 7 July 2021

Published online: 20 July 2021

\section{References}

1. Milos R-I, Moritz T, Bernathova M, Amann G, Panotopoulos J, NoebauerHuhmann I-M, Bodner G (2015) Superficial desmoid tumors: MRI and ultrasound imaging characteristics. Eur J Radiol 84(11):2194-2201. https:// doi.org/10.1016/j.ejrad.2015.08.012

2. Martínez Trufero J, Pajares Bernad I, Torres Ramón I, Hernando Cubero J, Pazo Cid R (2017) Desmoid-type fibromatosis: who, when, and how to treat. Curr Treat Options Oncol 18(5):29. https://doi.org/10.1007/s11 864-017-0474-0

3. Garcia-Ortega DY, Martín-Tellez KS, Cuellar-Hubbe M, Martínez-Said H, Álvarez-Cano A, Brener-Chaoul M, Alegría-Baños JA, Martínez-Tlahuel JL (2020) Desmoid-type fibromatosis. Cancers (Basel) 12(7):1851. https://doi. org/10.3390/cancers12071851

4. Ganeshan D, Amini B, Nikolaidis P, Assing M, Vikram R (2019) Current update on desmoid fibromatosis. J Comput Assist Tomogr 43(1):29-38. https://doi.org/10.1097/RCT.0000000000000790

5. Gounder MM, Thomas DM, Tap WD (2018) Locally aggressive connective tissue tumors. J Clin Oncol 36(2):202-209. https://doi.org/10.1200/JCO.2017. 75.8482
6. Gounder MM, Maddux L, Paty J, Atkinson TM (2020) Prospective development of a patient-reported outcomes instrument for desmoid tumors or aggressive fibromatosis. Cancer 126(3):531-539. https://doi.org/1 $0.1002 /$ cncr.32555

7. Lee S-H, Lee H-K, Song J-S, Jeong H-S (2012) Chest wall fibromatosis in the axilla. Arch Plast Surg 39(2):175-177. https://doi.org/10.5999/aps.2012.39.2.1 75

8. Braschi-Amirfarzan M, Keraliya AR, Krajewski KM, Tirumani SH, Shinagare AB, Hornick JL, Baldini EH, George S, Ramaiya NH, Jagannathan JP (2016) Role of imaging in management of desmoid-type fibromatosis: a primer for radiologists. Radiographics 36(3):767-782. https://doi.org/10.1148/rg.20161 50153

9. Zeitoun R, Khafagy SM, Mahmoud IH, El-Wahab NMA (2020) Radiological evaluation of deep soft tissue fibromatosis, the characteristic MR criteria on conventional and corresponding diffusion-weighted images. EJRNM 51(1). https://doi.org/10.1186/s43055-019-0097-5

10. Silva Filho AF, Alves JCRR, Portugal EH, Fonseca RPL, Almeida ACM, Pereira NA, Silva FL, Calsavara EF, Andrade Filho JS (2017) Aggressive fibromatosis (desmoid tumor) associated with breast implant: literature review and presentation of three new cases. Rev Bras Cir Plást 32(3):361-371. https:// doi.org/10.5935/2177-1235.2017RBCP0060

\section{Publisher's Note}

Springer Nature remains neutral with regard to jurisdictional claims in published maps and institutional affiliations.

\section{Submit your manuscript to a SpringerOpen ${ }^{\circ}$ journal and benefit from:}

- Convenient online submission

- Rigorous peer review

- Open access: articles freely available online

High visibility within the field

- Retaining the copyright to your article

Submit your next manuscript at $\boldsymbol{\nabla}$ springeropen.com 